\title{
Front Matter: Volume 8432
}

, "Front Matter: Volume 8432," Proc. SPIE 8432, Semiconductor Lasers and Laser Dynamics V, 843201 (30 May 2012); doi: 10.1117/12.979313

SPIE. Event: SPIE Photonics Europe, 2012, Brussels, Belgium 


\section{PROCEEDINGS OF SPIE}

\section{Semiconductor Lasers and Laser Dynamics V}

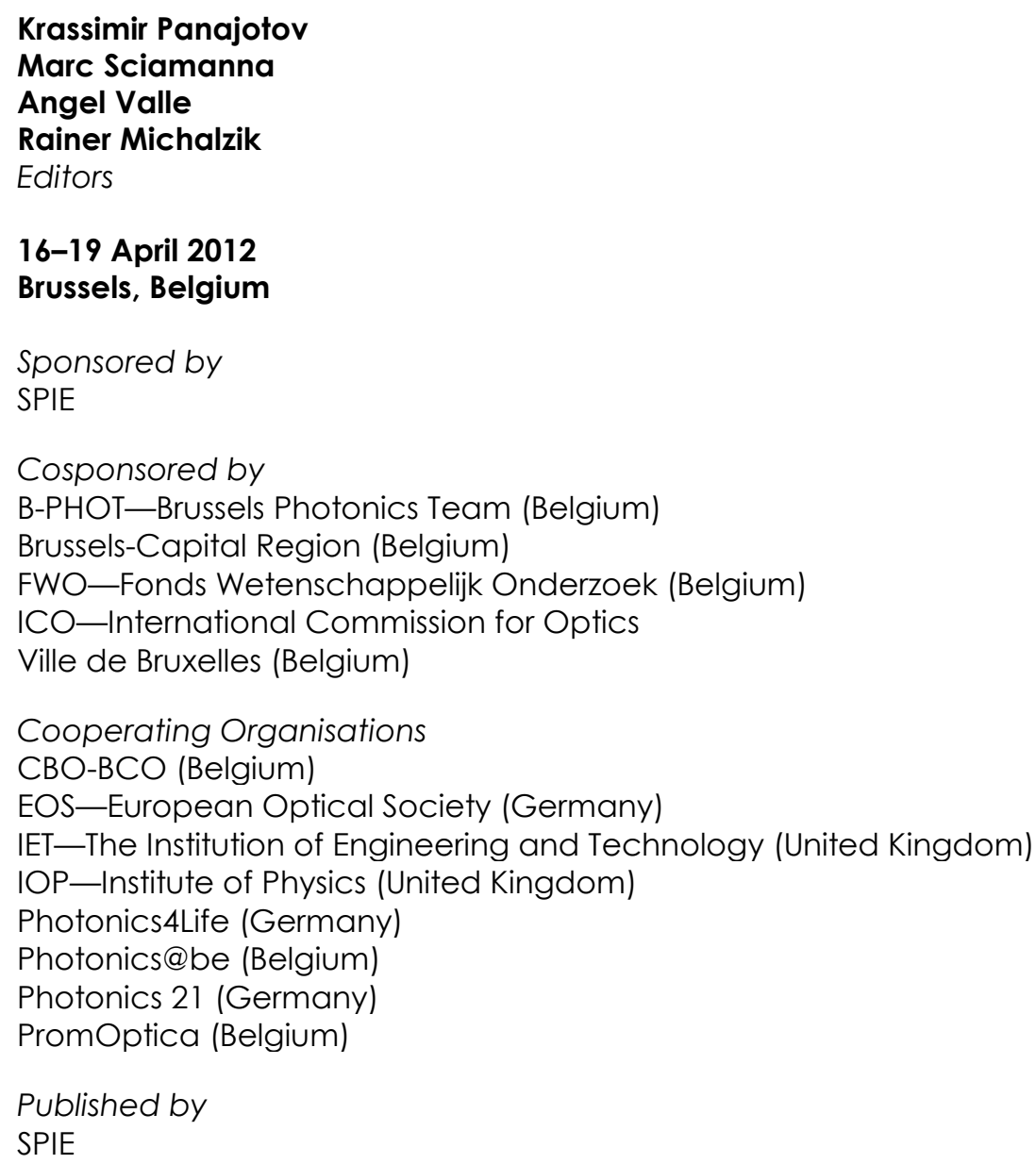


The papers included in this volume were part of the technical conference cited on the cover and title page. Papers were selected and subject to review by the editors and conference program committee. Some conference presentations may not be available for publication. The papers published in these proceedings reflect the work and thoughts of the authors and are published herein as submitted. The publisher is not responsible for the validity of the information or for any outcomes resulting from reliance thereon.

Please use the following format to cite material from this book:

Author(s), "Title of Paper," in Semiconductor Lasers and Laser Dynamics V, edited by

Krassimir Panajotov, Marc Sciamanna, Angel Valle, Rainer Michalzik, Proceedings of SPIE Vol. 8432 (SPIE, Bellingham, WA, 2012) Article CID Number.

ISSN 0277-786X

ISBN 9780819491244

Published by

SPIE

P.O. Box 10, Bellingham, Washington 98227-0010 USA

Telephone +1 3606763290 (Pacific Time) · Fax +1 3606471445

SPIE.org

Copyright (C) 2012, Society of Photo-Optical Instrumentation Engineers

Copying of material in this book for internal or personal use, or for the internal or personal use of specific clients, beyond the fair use provisions granted by the U.S. Copyright Law is authorized by SPIE subject to payment of copying fees. The Transactional Reporting Service base fee for this volume is $\$ 18.00$ per article (or portion thereof), which should be paid directly to the Copyright Clearance Center (CCC), 222 Rosewood Drive, Danvers, MA 01923. Payment may also be made electronically through CCC Online at copyright.com. Other copying for republication, resale, advertising or promotion, or any form of systematic or multiple reproduction of any material in this book is prohibited except with permission in writing from the publisher. The CCC fee code is 0277-786X/12/\$18.00.

Printed in the United States of America.

Publication of record for individual papers is online in the SPIE Digital Library.

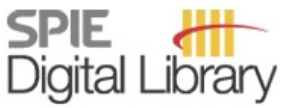

SPIEDigitalLibrary.org

Paper Numbering: Proceedings of SPIE follow an e-First publication model, with papers published first online and then in print and on CD-ROM. Papers are published as they are submitted and meet publication criteria. A unique, consistent, permanent citation identifier (CID) number is assigned to each article at the time of the first publication. Utilization of CIDs allows articles to be fully citable as soon as they are published online, and connects the same identifier to all online, print, and electronic versions of the publication. SPIE uses a six-digit CID article numbering system in which:

- The first four digits correspond to the SPIE volume number.

- The last two digits indicate publication order within the volume using a Base 36 numbering system employing both numerals and letters. These two-number sets start with $00,01,02,03,04$, $05,06,07,08,09,0 A, 0 B \ldots 0 Z$, followed by 10-1Z, 20-2Z, etc.

The CID number appears on each page of the manuscript. The complete citation is used on the first page, and an abbreviated version on subsequent pages. Numbers in the index correspond to the last two digits of the six-digit CID number. 


\section{Contents}

xi Conference Committee

\section{SESSION 1 VCSELS I}

843202 High-speed VCSELs for energy efficient computer interconnects (Invited Paper) [8432-01] P. Moser, P. Wolf, Technische Univ. Berlin (Germany); J. A. Lott, Vertically Integrated Systems GmbH (Germany); G. Larisch, Technische Univ. Berlin (Germany); A. Payusov, Technische Univ. Berlin (Germany) and St. Petersburg Academic Univ. (Russian Federation); A. Mutig, W. Unrau, Technische Univ. Berlin (Germany); N. N. Ledentsov, Vertically Integrated Systems GmbH (Germany); W. Hofmann, Technische Univ. Berlin (Germany); D. Bimberg, Technische Univ. Berlin (Germany) and King Abdulaziz Univ. (Saudi Arabia)

843203 New standards in high-speed and tunable long wavelength VCSELs (Invited Paper) [8432-02]

T. Gruendl, M. Mueller, M.-C. Amann, Technische Univ. Muenchen (Germany)

843204 Hybrid integration approach of VCSELs for miniaturized optical deflection of microparticles [8432-03]

A. Bergmann, N. I. Khan, J. A. Martos Calahorro, D. Wahl, R. Michalzik, Ulm Univ. (Germany)

843205 Transverse mode and polarization characteristics of AIGalnP-based VCSELs with integrated multiple oxide apertures [8432-04]

S. Weidenfeld, H. Niederbracht, M. Eichfelder, M. Jetter, P. Michler, Univ. of Stuttgart (Germany)

843206 VCSELs with two-sided beam emission for pressure sensor applications [8432-05]

B. Fischer, Vienna Univ. of Technology (Austria); A. Strodl, A. Hein, Univ. Ulm (Germany):

E. Wintner, Vienna Univ. of Technology (Austria); R. Michalzik, Univ. Ulm (Germany)

\section{SESSION 2 VCSELS II}

843209 Red AIGalnP-VECSEL emitting at around $665 \mathrm{~nm}$ : strain compensation and performance comparison of different epitaxial designs [8432-73]

T. Schwarzbäck, H. Kahle, M. Jetter, P. Michler, Univ. of Stuttgart (Germany)

8432 OA Three-dimensional simulation of 1300-nm AIGalnAs VCSEL arrays [8432-08]

T. Czyszanowski, M. Dems, M. Wasiak, R. P. Sarzala, Technical Univ. of Lodz (Poland)

8432 OB Polarization mode structure in long-wavelength wafer-fused vertical-cavity surface-emitting lasers (Best Student Paper Award) [8432-09]

N. Volet, V. lakovlev, A. Sirbu, Ecole Polytechnique Fédérale de Lausanne (Switzerland); A. Caliman, G. Suruceanu, A. Mereuta, Beam Express S.A. (Switzerland); E. Kapon, Ecole Polytechnique Fédérale de Lausanne (Switzerland) 
8432 OC Electro-optically modulated coupled-cavity VCSELs: electrical design optimization for high-speed operation [8432-10]

M. Zujewski, H. Thienpont, Vrije Univ. Brussel (Belgium); K. Panajotov, Vrije Univ. Brussel

(Belgium) and Institute of Solid State Physics (Bulgaria)

\section{SESSION 3 QUANTUM DOT LASERS I}

8432 OG Quantum dot microlasers with external feedback: a chaotic system close to the quantum limit [8432-14]

F. Albert, C. Hopfmann, C. Schneider, S. Höfling, L. Worschech, M. Kamp, W. Kinzel,

A. Forchel, S. Reitzenstein, Julius-Maximilians-Univ. Würzburg (Germany) I. Kanter, Bar-Ilan Univ. (Israel)

$8432 \mathrm{OH} \quad$ Intra-cavity absorber photocurrent characteristics of a quantum dot laser emitting on two emission-states: experiment and simulation [8432-15]

S. Brever, Technische Univ. Darmstadt (Germany); M. Rossetti, Politecnico di Torino (Italy); L. Drzewietzki, Technische Univ. Darmstadt (Germany); I. Montrosset, Politecnico di Torino (Italy); M. Hopkinson, Univ. of Sheffield (United Kingdom); W. Elsässer, Technische Univ. Darmstadt (Germany)

\section{SESSION 4 QUANTUM CASCADE LASERS I}

8432 Ol High performance GaAs/AIGaAs quantum cascade lasers: optimization of electrical and thermal properties (Invited Paper) [8432-16]

M. Bugajski, K. Kosiel, A. Szerling, P. Karbownik, K. Pierściński, D. Pierścińska, Institute of Electron Technology (Poland); G. Hałdaś, A. Kolek, Rzeszów Univ. of Technology (Poland)

8432 OL Facet reflectivity reduction of quantum cascade lasers by tilted facets [8432-19] S. I. Ahn, C. Schwarzer, S. Kalchmair, R. Gansch, D. Ristanic, T. Zederbaver, P. Reiniger, H. Detz, A. M. Andrews, W. Schrenk, G. Strasser, Vienna Univ. of Technology (Austria)

8432 OM Experimental analysis of thermal properties of AIGaAs/GaAs quantum cascade lasers [8432-20]

K. Pierściński, D. Pierścińska, M. Iwińska, K. Kosiel, A. Szerling, P. Karbownik, M. Bugajski, Institute of Electron Technology (Poland)

\section{SESSION 5 QUANTUM CASCADE LASERS II}

$8432 \mathrm{ON} \quad$ Room temperature continuous wave interband cascade lasers for gas sensing (Invited Paper) [8432-21]

S. Höfling, R. Weih, A. Baver, M. Kamp, A. Forchel, Julius-Maximilians-Univ. Würzburg (Germany)

843200 Near-field characteristics of broad area diode lasers during catastrophic optical damage failure [8432-22]

M. Hempel, J. W. Tomm, Max-Born-Institut (Germany); M. Baeumler, H. Konstanzer, Fraunhofer Institut für Angewandte Festkörperphysik (Germany); J. Mukherjee, Univ. of Surrey (United Kingdom); T. Elsaesser, Max-Born-Institut (Germany) 
$84320 Q \quad$ Spatial "rocking" for improving the spatial quality of the beam of broad area semiconductor lasers [8432-24]

M. Radziunas, Weierstrass Institute (Germany); K. Staliunas, Univ. Politècnica de Catalunya (Spain) and Institucio Catalana de Reserca i Estudis Avancats (Spain)

8432 OR Narrow linewidth discrete mode laser diodes at $1550 \mathrm{~nm}$ [8432-25]

J. O'Carroll, Eblana Photonics Ltd. (Ireland) and Dublin City Univ. (Ireland); R. Phelan, B. Kelly,

D. Byrne, Eblana Photonics Ltd. (Ireland); F. Smyth, B. Cardiff, P. M. Anandarajah, L. P. Barry,

Dublin City Univ. (Ireland)

\section{SESSION 6 SEMICONDUCTOR LASERS}

8432 OT Laterally-coupled high power GaSb distributed feedback lasers fabricated by nanoimprint lithography at $2 \mu \mathrm{m}$ wavelength [8432-27]

K. Haring, J. Paajaste, R. Koskinen, S. Suomalainen, J. Viheriälä, A. Laakso, T. Niemi, M. Guina, Tampere Univ. of Technology (Finland)

8432 OV Narrow linewidth $1120 \mathrm{~nm}$ GalnAs/GaAs VECSEL for cooling $\mathbf{M g}^{+}$ions [8432-29]

S. Ranta, T. Leinonen, M. Tavast, Tampere Univ. of Technology (Finland); R. Epstein, Areté Associates (United States); M. Guina, Tampere Univ. of Technology (Finland)

8432 OW Frequency doubled AIGalnP-VECSEL with high output power at $331 \mathrm{~nm}$ and a large wavelength tuning range in the UV [8432-30]

T. Schwarzbäck, H. Kahle, M. Jetter, P. Michler, Univ. of Stuttgart (Germany)

\section{SESSION 7 MODE-LOCKING}

8432 OY Passively mode-locked $1 \mathrm{GHz}$ MOPA system generating sub-500-fs pulses after external compression [8432-32]

T. Ulm, F. Harth, Photonik-Zentrum Kaiserslautern e.V. (Germany); A. Klehr, G. Erbert, Ferdinand-Braun-Institut (Germany); J. L'huillier, Photonik-Zentrum Kaiserslautern e.V. (Germany)

843210 Conversion between optical ASK and optical FSK using nonlinear dynamics of semiconductor lasers [8432-34]

S.-K. Hwang, National Cheng Kung Univ. (Taiwan); S.-C. Chan, City Univ. of Hong Kong

(Hong Kong, China); S.-L. Lin, C.-H. Chu, Y.-H. Hung, National Cheng Kung Univ. (Taiwan)

\section{SESSION 8 TIME DELAY SYSTEMS}

843212 Theoretical analysis of a multi-stripe laser array with external off-axis feedback [8432-36] A. Pimenov, Weierstrass Institute (Germany); G. Kozyreff, Univ. Libre de Bruxelles (Belgium); V. Z. Tronciu, Weierstrass Institute (Germany) and Technical Univ. of Moldova (Moldova);

A. G. Vladimirov, Weierstrass Institute (Germany)

843213 Low-frequency fluctuations in a laser diode with phase-conjugate feedback [8432-37] A. Karsaklian Dal Bosco, D. Wolfersberger, M. Sciamanna, Supélec (France) 
843214 Photonic single nonlinear-delay dynamical node for information processing [8432-38] S. Ortín, CSIC-Univ. de Cantabria (Spain); D. San-Martín, Predictia Intelligent Data Solutions (Spain); L. Pesquera, J. M. Gutiérrez, CSIC-Univ. de Cantabria (Spain)

843215 Dynamical properties of two delay-coupled lasers: on spectra, correlations, and synchronisation [8432-39]

O. D'Huys, Univ. Würzburg (Germany) and Vrije Univ. Brussel (Belgium); L. Lean, Vrije Univ. Brussel (Belgium); R. Vicente, Max-Planck Institut for Brain Research (Germany) and Frankfurt Institute for Advanced Studies (Germany); J. Danckaert, Vrije Univ. Brussel (Belgium); I. Fischer, Instituto de Fisica Interdisciplinar y Sistemas Complejos, Univ. de les Illes Balears-CSIC (Spain)

\section{SESSION 9 NANOLASER AND OPTICAL INJECTION}

843219 Mapping transients in the nonlinear dynamics of an optically injected VCSEL [8432-43] J. P. Toomey, D. M. Kane, Macquarie Univ. (Australia); K. Schires, Univ. of Essex (United Kingdom); C. Nichkawde, Macquarie Univ. (Australia); A. Hurtado, I. D. Henning, M. J. Adams, Univ. of Essex (United Kingdom)

\section{SESSION 10 SEMICONDUCTOR LASERS AND OPTICAL INJECTION}

8432 1B Deterministic and stochastic dynamics of linear polarizations emitted by single-mode VCSELs subject to orthogonal optical injection [8432-45]

P. Pérez, A. Quirce, CSIC-Univ. de Cantabria (Spain) and Univ. de Cantabria (Spain);

A. Valle, L. Pesquera, CSIC-Univ. de Cantabria (Spain)

$84321 \mathrm{C}$ Polarization switching of transverse modes in VCSELs subject to two-frequency orthogonal optical injection [8432-46]

A. Quirce, CSIC-Univ. de Cantabria (Spain) and Univ. de Cantabria (Spain); A. Valle, CSIC-Univ. de Cantabria (Spain); H. Lin, Y. Zhang, D. W. Pierce, Bates College (United States)

8432 1D Delay induces motion of multipeak localized structures in cavity semiconductors [8432-47] E. Averlant, Univ. Libre de Bruxelles (Belgium) and Vrije Univ. Brussel (Belgium); M. Tlidi, Univ. Libre de Bruxelles (Belgium); A. G. Vladimirov, Weierstrass Institute for Applied Analysis and Stochastics (Germany); H. Thienpont, Vrije Univ. Brussel (Belgium); K. Panajotov, Vrije Univ. Brussel (Belgium) and Institute of Solid State Physics (Bulgaria)

$84321 \mathrm{E}$ Dynamics accompanying polarization switching in vertical-cavity surface-emitting lasers [8432-48]

M. Virte, Supélec (France) and Vrije Univ. Brussel (Belgium); K. Panajotov, Vrije Univ. Brussel (Belgium) and Institute of Solid State Physics. (Bulgaria); M. Sciamanna, Supélec (France)

8432 if High performance identical layer InGaAIAs-MQW 1300nm electroabsorption-modulated DFB-lasers for $4 \times 25 \mathrm{Gbit} / \mathrm{s}$ [8432-80]

H. Klein, C. Bornholdt, G. Przyrembel, A. Sigmund, W.-D. Molzow, M. Moehrle, Fraunhofer Institute for Telecommunications, Heinrich-Hertz Institute (Germany) 
$84321 G$ True photonic band-gap in vertical-cavity surface-emitting lasers [8432-50]

M. Dems, Technical Univ. of Lodz (Poland); K. Panajotov, Vrije Univ. Brussel (Belgium) and Institute of Solid State Physics (Bulgaria)

$8432 \mathrm{lH} \quad$ Simulation of 1550-nm diamond VECSEL with high contrast grating [8432-51]

J. Walczak, T. Czyszanowski, M. Dems, R. P. Sarzala, A. Sokol, M. Wasiak, Technical Univ. of Lodz (Poland); V. Iakovlev, Ecole Polytechnique Fédérale de Lausanne (Switzerland)

$843211 \quad$ Semiconductor ring lasers as optical neurons [8432-52]

W. Coomans, L. Gelens, L. Mashal, S. Beri, G. Van der Sande, J. Danckaert, G. Verschaffelt, Vrije Univ. Brussel (Belgium)

\section{SESSION 12 QUANTUM DOT LASERS II}

$84321 \mathrm{~J} \mathrm{Many-body} \mathrm{effects} \mathrm{and} \mathrm{self-contained} \mathrm{phase} \mathrm{dynamics} \mathrm{in} \mathrm{an} \mathrm{optically} \mathrm{injected}$ quantum-dot laser [8432-53]

B. Lingnau, K. Lüdge, Technische Univ. Berlin (Germany); W. W. Chow, Sandia National Labs. (United States); E. Schöll, Technische Univ. Berlin (Germany)

$84321 \mathrm{~L}$ Simultaneous multi-state stimulated emission in quantum dot lasers: experiment and analytical approach [8432-55]

V. V. Korenev, St. Petersburg Academic Univ. (Russian Federation); A. V. Savelyev, St. Petersburg Academic Univ. (Russian Federation) and St. Petersburg State Polytechnical Univ. (Russian Federation); A. E. Zhukov, St. Petersburg Academic Univ. (Russian Federation) and St. Petersburg State Polytechnical Univ. (Russian Federation) and loffe Physico-Technical Institute (Russian Federation); A. V. Omelchenko, St. Petersburg Academic Univ. (Russian Federation) and St. Petersburg State Polytechnical Univ. (Russian Federation); M. V. Maximov, St. Petersburg Academic Univ. (Russian Federation) and loffe Physico-Technical Institute (Russian Federation); Y. M. Shernyakov, loffe Physico-Technical Institute (Russian Federation)

\section{POSTER SESSION}

$84321 \mathrm{M}$ Theoretical study about the gain in indirect bandgap semiconductor acousto-optical cavities with simultaneous photon and phonon confinement [8432-57] J. M. Escalante Fernandez, A. Martínez, Univ. Politècnica de València (Spain)

$84321 \mathrm{P}$ Self-consistent simulation of mid-IR quantum cascade lasers based on rate equation approach [8432-61]

P. Karbownik, M. Bugajski, Institute of Electron Technology (Poland)

$84321 Q \quad$ Nonlinear dynamics in directly modulated semiconductor ring lasers [8432-62]

S. T. Kingni, Univ. of Yaoundé I (Cameroon) and Vrije Univ. Brussel (Belgium);

G. Van der Sande, L. Gelens, J. Danckaert, Vrije Univ. Brussel (Belgium)

$8432 \mathrm{IR}$ Experimental and numerical study of square wave oscillations due to asymmetric optical feedback in semiconductor ring lasers [8432-63]

L. Mashal, G. Van der Sande, L. Gelens, S. Beri, Vrije Univ. Brussel (Belgium); T. Erneux, Univ. Libre de Bruxelles (Belgium); J. Danckaert, G. Verschaffelt, Vrije Univ. Brussel (Belgium) 
8432 is Electrical and optical characterisation of mid-IR GaAs/AIGaAs quantum cascade lasers [8432-64]

D. Pierścińska, K. Pierściński, M. Iwińska, K. Kosiel, A. Szerling, P. Karbownik, M. Bugajski, Institute of Electron Technology (Poland)

$84321 \mathrm{~T} \quad$ Low timing jitter $\mathbf{4 0} \mathbf{~} \mathbf{~ b}$ /s all-optical clock recovery based on an amplified feedback laser diode [8432-65]

C. Chen, J. Qiu, L. Zhao, Institute of Semiconductors (China); J. Wu, Beijing Univ. of Posts and Telecommunications (China); C. Lou, Tsinghua Univ. (China); W. Wang, Institute of Semiconductors (China)

$84321 \mathrm{U}$ Interaction between laser beams FP and DFB laser diodes [8432-66]

V. Vasinek, J. Latal, P. Koudelka, P. Siska, J. Vitasek, K. Witas, Technical Univ. of Ostrava (Czech Republic)

$84321 \mathrm{~V}$ Optical injection locking of polarization modes and spatial modes in single-aperture VCSELs and VCSEL arrays emitting at $1.3 \mu \mathrm{m}$ [8432-67]

E. Lamothe, C. Long, Ecole Polytechnique Fédérale de Lausanne (Switzerland); A. Mereuta, A. Caliman, Beam Express S.A. (Switzerland); A. Sirbu, V. Iakovlev, Ecole Polytechnique Fédérale de Lausanne (Switzerland); G. Suruceanu, Beam Express S.A. (Switzerland); E. Kapon, Ecole Polytechnique Fédérale de Lausanne (Switzerland)

8432 1X 850nm VCSEL with a liquid crystal overlay [8432-69]

V. M. Nair, Vrije Univ. Brussel (Belgium); K. Panajotov, Vrije Univ. Brussel (Belgium) and Institute of Solid State Physics (Bulgaria); M. Petrov, Institute of Solid State Physics (Bulgaria); H. Thienpont, Vrije Univ. Brussel (Belgium); Y. Xie, J. Beeckman, K. Neyts, Ghent Univ. (Belgium)

$84321 \mathrm{Y} \quad$ Extended synchronization resulting from resonant phase and intensity dynamics in a dual-polarization laser [8432-70]

J. Thévenin, M. Romanelli, M. Brunel, M. Vallet, Institut de Physique de Rennes, CNRS, Univ. de Rennes 1 (France); T. Erneux, Univ. Libre de Bruxelles (Belgium)

$84321 \mathrm{D}$ Discrete mode laser diodes for FTTH/PON applications up to $10 \mathrm{Gbit} / \mathrm{s}$ [8432-71]

J. O'Carroll, Eblana Photonics Ltd. (Ireland) and Dublin City Univ. (Ireland); R. Phelan, B. Kelly, D. Byrne, Eblana Photonics Ltd. (Ireland); S. Latkowski, P. M. Anandarajah, L. P. Barry, Dublin City Univ. (Ireland)

843222 Improving beam quality in broad area semiconductor amplifiers [8432-76]

R. Herrero, M. Botey, N. P. Kumar, K. Staliunas, Univ. Politècnica de Catalunya (Spain)

843225 Carrier escape from ground state and non-zero resonance frequency at low bias powers for semiconductor quantum-dot lasers [8432-79]

C. Wang, Univ. Européenne de Bretagne, INSA, CNRS FOTON (France); F. Grillot, Univ. Européenne de Bretagne, INSA, CNRS FOTON (France) and Telecom Paristech, Ecole Nationale Supérieure des Télécommunications, CNRS LTCI (France); J. Even, Univ. Européenne de Bretagne, INSA, CNRS FOTON (France) 
843226 Self-mixing in VCSELs for multi-parametric sensing applications: theory and experiment [8432-81]

L. Columbo, Istituto di Fotonica e Nanotecnologie, CNR, Univ. degli Studi di Bari (Italy) and Univ. degli Studi dell'Insubria (Italy); F. De Lucia, M. Brambilla, M. Dabbicco, G. Scamarcio, Istituto di Fotonica e Nanotecnologie, CNR, Univ. degli Studi di Bari (Italy)

Author Index 
Downloaded From: https://www.spiedigitallibrary.org/conference-proceedings-of-spie on 26 Apr 2023

Terms of Use: https://www.spiedigitallibrary.org/terms-of-use 


\title{
Conference Committee
}

\author{
Symposium Chairs
}

Francis Berghmans, Vrije Universiteit Brussel (Belgium)

Ronan Burgess, European Commission (Belgium)

Jürgen Popp, Institut für Photonische Technologien e.V. (Germany)

Peter Hartmann, SCHOTT AG (Germany)

Honorary Symposium Chair

Hugo Thienpont, Vrije Universiteit Brussel (Belgium)

Conference Chairs

Krassimir Panajotov, Vrije Universiteit Brussel (Belgium)

Marc Sciamanna, Supélec (France)

Angel Valle, Universidad de Cantabria (Spain)

Rainer Michalzik, Universität Ulm (Germany)

\section{Programme Committee}

Sylvain Barbay, Centre National de la Recherche Scientifique (France)

Dieter Bimberg, Technische Universität Berlin (Germany)

Weng W. Chow, Sandia National Laboratories (United States)

Kent D. Choquette, University of Illinois at Urbana-Champaign (United States)

Wolfgang E. Elsaesser, Technische Universität Darmstadt (Germany)

Anders Larsson, Chalmers University of Technology (Sweden)

Jesper Moerk, Technical University of Denmark (Denmark)

Jerome V. Moloney, College of Optical Sciences, The University of Arizona (United States)

Wlodzimierz Nakwaski, Technical University of Lodz (Poland)

K. Alan Shore, Bangor University (United Kingdom)

Anne C. Tropper, University of Southampton (United Kingdom)

Atsushi Uchida, Saitama University (Japan)

Joachim Wagner, Fraunhofer-Institut für Angewandte Festkörperphysik (Germany)

Sebastian Wieczorek, The University of Exeter (United Kingdom) 


\section{Session Chairs}

1 VCSELS I

Krassimir Panajotov, Vrije Universiteit Brussel (Belgium)

2 VCSELS II

Rainer Michalzik, Universität Ulm (Germany)

3 Quantum Dot Lasers I

Philip Moser, Technische Universitäł Berlin (Germany)

4 Quantum Cascade Lasers I

Sven Höfling, Julius-Maximilians-Universität Würzburg (Germany)

5 Quantum Cascade Lasers II

Maciej Bugajski, Institute of Electron Technology (Poland)

6 Semiconductor Lasers

Weng W. Chow, Sandia National Laboratories (United States)

$7 \quad$ Mode-Locking

Stefan Brever, Technische Universität Darmstadt (Germany)

8 Time Delay Systems

Angel Valle, Universidad de Cantabria (Spain)

$9 \quad$ Nanolaser and Optical Injection

Marc Sciamanna, Supélec (France)

10 Semiconductor Lasers and Optical Injection

Sylvain Barbay, Centre National de la Recherche Scientifique (France)

11 Semiconductor Lasers

Andrei G. Vladimirov, Weierstrass-Institut für Angewandte Analysis und Stochastik (Germany)

12 Quantum Dot Lasers II

Krassimir Panajotov, Vrije Universiteit Brussel (Belgium) 